\title{
Two-way and One-way Vacuum Speed of Light under the Membrane Paradigm
}

\author{
Stefan von Weber ${ }^{1 *}$ and Alexander von Eye ${ }^{2}$ \\ ${ }^{1}$ Faculty Medical and Life Sciences, Furtwangen University, Jakob-Kienzle-Strasse 14, 78054 \\ Villingen-Schwenningen, Germany. \\ ${ }^{2}$ Department of Psychology, Michigan State University, Psychology Building, 318 Physics Rm 262, \\ East Lansing, MI 48824, USA.
}

Authors' contributions

This work was carried out in collaboration between both authors. Author SVW wrote the first draft of the manuscript. Both authors together managed the literature search, the mathematical formulation and read and approved the final manuscript.

Article Information

DOI: $10.9734 / \mathrm{PSIJ} / 2017 / 32988$

Editor(s):

(1) Aleksey Anatolievich Zakharenko, The International Institute of Zakharenko Waves (IIZWs), Krasnoyarsk, Siberia, Russia.

(2) Christian Brosseau, Department of Physics, Université de Bretagne Occidentale, France.

Reviewers:

(1) Sanjay Sastry, CF Cosmological Inst., Orlando, Florida, USA.

(2) Francisco Bulnes, Tecnológico de Estudios Superiores de Chalco, Mexico.

(3) El-Nabulsi Ahmad Rami, Cheju National University, South Korea.

(4) Bernard Lavenda, Universita degli Studi, Camerino, Italy. (5) Alejandro Gutiérrez-Rodríguez, Universidad Autónoma de Zacatecas, México.

(6) Stanislav Fisenko, Moscow State Linguistic University, Russia. Complete Peer review History: http://www.sciencedomain.org/review-history/19837

Original Research Article

Received 26 ${ }^{\text {th }}$ March 2017 Accepted $27^{\text {th }}$ June 2017 Published $3^{\text {rd }}$ July 2017

\section{ABSTRACT}

The discovery of cosmic microwave background radiation (CMB) by Wilson and Penzias defines a rest frame in the sense of Newton's absolute space. This fact is one of the reasons why brane worlds moved into the focus of interest. Membrane theory (CM) uses the cosmological model of a 4-dimensional thin membrane, expanding in hyperspace. A homogeneous vector field acts perpendicularly from outside onto the membrane and causes, this way, curvature of space and gravitation. The membrane defines an absolute Newtonian space, and forces small changes of Special Relativity (SR). The most important difference between CM and SR is the introduction of a cross contraction of moving bodies.

Photons travel always and only in the rest inertial reference frame. There is no difference between two-way and one-way speed of light. Far away from masses photons move with constant speed $c$. 
The rest inertial reference frame is defined by the absence of the dipole of the cosmic microwave background radiation (CMB), caused by the Doppler-effect. The speed of light is anisotropic in each inertial reference frame that is in relative motion with respect to the rest frame. Although the speed of light is constant in the vacuum, it can be measured on Earth only with a systematic error of \pm $36.9 \mathrm{~m} / \mathrm{s}$. The reason is that the flow of time of the clocks on the Earth is not constant.

The measurement of the one-way speed of light is a special issue. Although the speed of light is anisotropic in each moving inertial reference frame, the time transformation hides this fact nearly perfectly. A co-moving observer registers the arrival of the light signal after a time interval which suggests that speed of light is always $c$. To obtain this result, it is sufficient to accept the existence of a rest inertial reference frame and the transformation of time given by FitzGerald and Lorentz.

Keywords: Special relativity; time dilation; length contraction; cross contraction; speed of light; oneway; cosmic microwave background radiation (CMB); dipole; Cosmic Membrane Theory (CM).

\section{INTRODUCTION}

One hundred and ten years after the publication of the theory of relativity by Albert Einstein, new scientific insights have been gathered that make it advisable to develop the theory of relativity further. In this regard, many consider the cosmic microwave background radiation (CMB) by Wilson and Penzias [1] the most important discovery, because CMB depicts, by its dipole character, the absolute motion of Earth in space.

All measurements of the speed of light use the two-way arrangement. The light travels to a mirror, is reflected and returns to its starting point where the clock is positioned. In contrast, the measurement of the one-way speed of light, that is, the measurement over a single distance without reflection, is more complicated. . Such a measurement needs, besides the knowledge of the exact distance, two synchronized clocks one clock at the starting point and another clock at the arrival point. Here, synchronization is the issue. We demonstrate in this paper that this synchronization is not possible in a simple manner in a moving inertial reference frame such as the Earth orbiting about the Sun. However, one needs the one-way arrangement, e.g., for the measurement of time of travel of neutrinos or gravitational waves. One way to react to the existence of an absolute space is to establish a brane world. Our aim was to find a cosmological model which is near to special and general relativity. Cosmic Membrane Theory (CM) uses the model of a 4-dimensional balloon (membrane) with a thin skin, expanding into hyperspace [2,3,4]. The 3-dimensional surface of the balloon (Newton's absolute space, membrane, space-time, quantum vacuum, or rest inertial reference frame) is our cosmos. A homogeneous vector field $[5,6,4]$ acts perpendicularly from outside onto the membrane, and causes the local curvature of the space which is otherwise the cause of gravitation and dark matter $[4,7,8,9]$.

Estimated conformity between $\mathrm{CM}$ and $\mathrm{SR}$ is nearly $100 \%$. The conformity concerns, e.g., Maxwell's equations, Fresnel's drag coefficient, Fizeau's experiment, aberration and Airy's experiment, Sagnac's effect, the atomic clock experiment of Haefele and Keating, the TroutonNoble experiment, and the Thomas precession of the electron. The only essential difference is the introduction of a cross contraction of moving bodies [10]. Cross contraction is the cause of the decrease of the cross section of accelerated particles, and, together with the contraction of length, cross contraction guarantees a constant path of light in each inertial reference frame. The ratio of length contraction and cross contraction is $\left(1-\beta^{2}\right)^{1 / 2}$, i.e., the same ratio as in FitzgeraldLorentz transformation.

Conformity between General Relativity (GR) and CM is also nearly $100 \%$. Here, the conformity concerns, e.g., light bending by stars and galaxies, Einstein rings, the time delay of radar signals grazing the edge of the Sun, gravitational red shift, gravitational time dilation of clocks, gravitational waves, and red shift caused by the expansion of the Universe. The few differences include the decrease of the speed of light and the increase of mass in strong gravitational fields. But here, thanks to Puthoff's "Polarizable Vacuum approach to GR" [11], CM and GR are also in agreement.

Besides a simpler derivation of Newton's law of attraction and a simpler explanation how gravity acts, the CM model gives an explanation of dark matter. Dark matter does not consist of particles, 
but is an effect of the homogeneous vector field acting on the curved membrane [7]. Because we find the curvature of space only in the neighborhood of baryonic matter, dark matter appears only together with it.

The negation of the frame-dragging effect by the $\mathrm{CM}$ model remains the only essential difference between CM and GR. In [9] the authors demonstrated that the result of the Gravity Probe $B$ experiment concerning the frame dragging is, with high probability, caused by the gravitation of Sun together with the motion of Earth in respect to the rest frame. Otherwise, the LAGEOS laser ranging stated a clear linear precession of the polar orbit as described by Ciufolini [12]. But there is a difference. The orbital planes of the Laser Geodynamics Satellites (LAGEOS) perform a precession as a whole. In the Gravity Probe $B$ experiment, only the spin axis of the gyroscope should perform the precession. We suppose that this difference has some deeper physical meaning, and should be the subject of future research. The speed of light in the vacuum is a constant, but it can be measured on Earth only with a systematic error. The reason is that the flow of time of the clocks on the Earth is not constant, which is caused by the relative motion of the Earth around the Sun. The authors predict a sine-shaped yearly variation with an amplitude of $36.9 \mathrm{~m} / \mathrm{s}$, and with the maximum reached in the summer for new measurements of the speed of light. This hypothesis is only apparently in contradiction to the very exact measurements of Evenson and Petersen in 1972 [13]. Evenson and Petersen replaced the measurement of the distance by a measurement of a frequency (and thus by a second measurement of time). The consequence is that a change in the time flow has no further influence on the measurement of the velocity. The handling of time by Evenson and Petersen amounts to the geocentric time system, and is in agreement with the definition of the speed of light as a constant.

This article is structured as follows: In Section 2, we refer to the fact, that the speed of light is not constant in all cases. In Section 3, we show the constancy of the speed of light for each inertial reference frame also under the paradigm of a membrane. In Section 4, we consider the time and the time transformation. In Section 5, we propose a new measurement of the speed of light. In Section 6, we consider one-way measurement of light and its implications. The article ends with a discussion (section 7) and conclusions (section 8).

\section{THE SPEED OF LIGHT}

The speed of light is a Natural phenomenon which aroused the interest of science over centuries. In 1676, the astronomer Ole Christensen Roemer was the first to measure the speed of light. Later, researchers obtained increasingly exact values, until, in 1973, the speed of light was established as Natural constant. The foundation for this act was the very exact measurement of the Boulder group under the direction of Ken Evenson [13].

The prediction of the cosmic micro-wave background radiation (CMBR) by Gamow, Doroshkevich and Novikov [14] and its discovery by Arno Penzias and Robert Wilson [1] with a clearly defined dipole $[15,16]$ support the hypothesis of an absolute space in the sense of Newton. Other names of the absolute space are space-time, rest inertial reference frame, quantum vacuum, or membrane. One can explain the dipole of the cosmic micro-wave background radiation as a Doppler-effect that is caused by the motion of the Earth in the rest inertial reference frame.

However, the speed of light is not constant for the entire space $[17,18,19,20,21,22]$. Already Einstein considered in 1911 the possibility that the speed of light could alter in the neighborhood of heavy masses. In 1957, R. H. Dicke suggested that the electric permittivity and the magnetic permeability, $\varepsilon_{0}$ and $\mu_{0}$ respectively, of the space-time can change. H. Puthoff [11] seized the suggestion and upgraded and modernized Dicke's theory. Puthoff showed that his "Polarizable Vacuum approach to GR" is in agreement with general relativity, but gives a deeper insight into the issue of space-time. Masses change not only the curvature of spacetime, but also its physical properties. One consequence of Puthoff's theory is that we have to exclude areas with strong gravitational fields, that is, areas in the neighborhood of stars or black holes, when we consider the vacuum speed of light.

\section{CONSTANCY OF THE TWO-WAY SPEED OF LIGHT}

Besides the principle of relativity, the constancy of the speed of light is one of the foundation pillars of special theory of relativity (SR). Increasingly exact experiments and measurements have confirmed the hypothesis 
that the vacuum speed of light, $\mathrm{c}$, is a fundamental physical constant. One of the fundamental principles of $S R$ is that $c$ is the same in all inertial reference frames.

The introduction of a cosmic membrane as an absolute space in the sense of Newton defines a specific inertial reference frame. This frame is that coordinate system in which cosmic microwave background radiation has no dipole [15]. The dipole is caused by the motion of the Earth relative to the background radiation and the connected Doppler effect. We name this special inertial reference frame the rest inertial reference frame. All other inertial reference frames are moving relative to the rest inertial reference frame with a constant speed of $v<c$. Naturally, we are unable to find a real inertial reference frame because each motion is accelerated by outer influences, as the rotation of the Earth, the gravitational force of the Sun, of the galaxy etc.

In this article, we show that the constancy of the speed of light remains valid for each inertial reference frame even when an absolute space is introduced. To this effect, we use a time and coordinate transformation which represents a minor alteration of the Fitz Gerald-Lorentz transformation. The most important change is the introduction of a cross contraction of moving bodies. However, the ratio of cross contraction and length contraction remains the same, as in the FitzGerald-Lorentz transformation [10].

Two of the fundamental principles of the membrane theory concerning the vacuum speed of light far from disturbing masses are:

1. Photons move always and only in the rest inertial reference frame.

2. Far away from masses, photons move with constant speed c.

In 1887, Michelson and Morley intended to prove the motion of the Earth in the resting ether, in their experiment with the interferometer. The negative result is known, and led to the theory of special relativity of Albert Einstein (1905). Other well known names in this context include FitzGerald (1884), Voigt (1887), Lorentz (1892), and Poincaré (1905).

Hendrik Lorentz formulated in 1892 the equations of his transformation which describe the changes in time and length when one changes from an inertial reference frame $\sigma$ to another inertial reference frame $\sigma$, one that is in relative motion with respect to $\sigma$. (In this article, we denote the rest inertial reference frame by $\sigma_{\mathrm{r}}$.) Lorentz' equations are symmetric, i.e., they describe the backward transformation as well. The equations of the FitzGerald-Lorentz transformation are part of the SR, and this theory is consistent with all experiments so far.

However, one disadvantage of the SR is its phenomenological nature. That is, as a descriptive theory, it is not responsive to the space-time fabric of the quantum vacuum. The SR abstracts from all physical properties of the space. However, the discovery of a natural rest inertial reference frame evokes questions concerning exactly these properties.

A second issue is that the time transformation was verified by many experiments, but not the length transformation. We can find this transformation only indirectly, and this is the subject of our interest. Von Weber and Onoochin [10] postulated a length contraction as well a cross contraction for moving bodies.

We show two plausible reasons for the introduction of the cross contraction. (1) The constancy of the electromagnetic forces of attraction and rejection conserves the energy of the lattice of a crystal no matter the speed $v$ of the inertial reference frame $\sigma^{\prime}$ of the crystal [10]. (2) It is known that the resolving power of collider experiments increases with the energy $E$ of the accelerated particles proportionally to $1 / E$, i.e., the diameters of the testing particles decrease with $1 / E$. The resolution accuracy can be described using the wavelength $\lambda$ of de Broglie,

$$
\lambda=\frac{h}{p}=\frac{h c}{\left(E^{2}-m_{0}^{2} c^{4}\right)^{1 / 2}} .
$$

When the speed $v$ of a particle is near the speed of light, the energy $E$ of the particle is directly proportional to the mass $m(v)=m_{0} /\left(1-v^{2} / c^{2}\right)^{1 / 2}, \quad$ and, thus,
$E \approx m_{0} /\left(1-v^{2} / c^{2}\right)^{1 / 2}$ or $\quad \lambda \approx\left(1-v^{2} / c^{2}\right)^{1 / 2}$. This relation is the cross contraction. That is, the contraction of the cross diameter that can be described by $y^{\prime}=y\left(1-v^{2} / c^{2}\right)^{1 / 2}$. The Michelson-Morley experiment [23] then leads necessarily to the length contraction 
$x^{\prime}=x\left(1-v^{2} / c^{2}\right)$. The time transformation remains unchanged [24], i.e., $\Delta t^{\prime}=\Delta t /\left(1-v^{2} / c^{2}\right)^{1 / 2}$. The time transformation was proven by numerous experiments, including, e.g., the one by Ives und Stilwell (1938) [25], or by experiences with the Global Positioning System (GPS).

In the following text, we denote, as usual, the quotient $v / c$ by $\beta$, i.e. $\beta=v / c$. The quotient $\beta$ is independent on the scales of length and time. To explain, we first observe the motion of a light signal in the moving inertial reference frame $\sigma$ '. The velocity $v$ of $\sigma^{\prime}$ with respect to the rest inertial reference frame $\sigma_{\mathrm{r}}$ can be measured in units of the rest inertial reference frame. Let the direction of $v$ be, without loss of generality, the positive $x$-direction. Let the quantity $y^{\prime}$ be a constant distance perpendicular to $x$, measured in units of the rest inertial reference frame. The light signal moves apparently in the moving inertial reference frame o' over the distance $y$, is reflected by a mirror, and returns to the starting point, the source of light. The true path of the light signal in the rest inertial reference frame is given in Fig. 3.1.

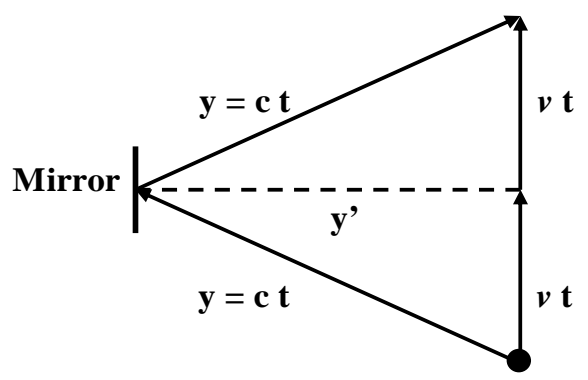

Light source

Fig. 3.1. Light path perpendicular to the direction of motion

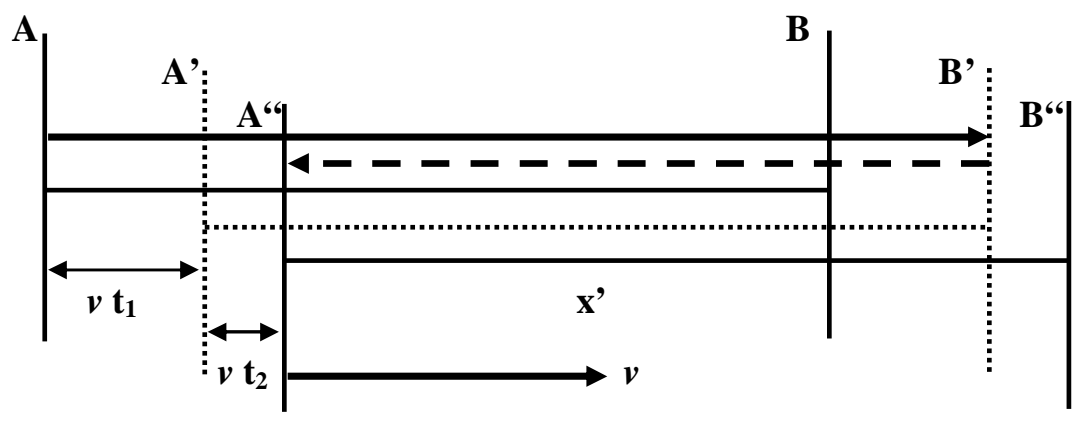

Fig. 3.2. Light path parallel to the direction of motion
From Fig. 3.1, it follows that

$$
y^{\prime}=\left((c t)^{2}-(v t)^{2}\right)^{1 / 2}
$$

or

$$
y^{\prime}=c t\left(1-\beta^{2}\right)^{1 / 2}=y\left(1-\beta^{2}\right)^{1 / 2} .
$$

In the rest inertial reference frame, we find $y=c$ $t$. Thus, Eq. (3.3) describes the cross-contraction of a body with the width $y$ when resting in the rest inertial reference frame $\sigma_{r}$, that, when accelerated to the velocity $v$, has the contracted width $y$ '. One issue of relativity is the choice of the scales. Unless otherwise declared, we use the rulers and clocks of the rest inertial reference frame $\sigma_{r}$. For example, when we write $y^{\prime}$ we denote a distance in the moving inertial reference frame $\sigma$ ' measured with the ruler of $\sigma_{r}$.

The derivation of the length contraction is more complicated. Here, the light path and the velocity $v$ of the motion of the moving inertial reference frame $\sigma$ ' are parallel. We show that the true distance covered by the light signal is the same as that of the rest inertial reference frame. Consider the length-contracted light path $x^{\prime}$ parallel to $v$. A light signal moves from point $A$ to point B over the moving distance $x^{\prime}$, is reflected by the co-moving mirror, and returns to the comoving starting point. The distance $x^{\prime}$ is contracted by length contraction, i.e., its current length differs from its length in the rest inertial reference frame $\sigma$. When the light signal reaches Point B', the whole distance $x$ ' has moved by the distance $c t_{1}$, i.e., the light path is extended. After reflection in point B', the light signal travels back and meets the starting point in its new position $A^{\prime \prime}$. The starting point has traveled the distance $\mathrm{Ct}_{2}$ with respect to $\mathrm{A}$ '. 
We find

$$
B^{\prime}-A=x^{\prime}+v t_{1}=c t_{1}
$$

and

$$
A^{\prime \prime}-B^{\prime}=x^{\prime}-v t_{2}=c t_{2}
$$

We divide Eq. (3.4) by $t_{1}$ and obtain $x^{\prime} / t_{1}=c-v$, and, in a similar manner, we obtain $x^{\prime} / t_{2}=c+v$ from Eq. (3.5). Now, we posit that the total run time, $t=2 x / c$, of the light signal for both parts of the path in the resting light distance $x$ does not change. We obtain

$$
t=t_{1}+t_{2}=\frac{x^{\prime}}{c-v}+\frac{x^{\prime}}{c+v}=\frac{2 x}{c}
$$

or

$$
\frac{x^{\prime}(c+v)+x^{\prime}(c-v)}{(c-v)(c+v)}=\frac{2 x^{\prime} c}{c^{2}-v^{2}}=\frac{2 x}{c}
$$

or

$$
x^{\prime}=x\left(c^{2}-v^{2}\right) / c^{2},
$$

or

$$
x^{\prime}=x\left(1-\beta^{2}\right) \text {, respectively. }
$$

Eq. (3.8) describes the length contraction of a body with length $x$ in the rest inertial reference frame $\sigma_{\mathrm{r}}$, that, when accelerated to velocity $v$, assumes the contracted length $x^{\prime}$.

Now, we show that the runtime $t$ of the light signal is constant for an arbitrary angle $\varphi$ between distance $A B$ and direction of the motion (direction of velocity $v$ ) of the moving inertial reference frame $\sigma$ '. Imagine a reflecting, hollow sphere with radius $R$. In the rest inertial reference frame $\sigma_{r}$, the hollow sphere reflects a light signal that originates in the center back to the center. The run time is $t=2 R / c$. Now, the hollow sphere is accelerated to speed $v$ and changes its spherical shape to the shape of an ellipsoid of revolution (spheroid). The minor axis (axis of revolution) is, because of the length contraction, $x^{\prime}=R\left(1-\beta^{2}\right)$. Let $y^{\prime}$ be one of the major axes.
Its length is, because of the cross contraction, $y^{\prime}=R\left(1-\beta^{2}\right)^{1 / 2}$

Consider Fig. 3.3. A source of light, $A$, travels with speed $v$ in the rest inertial reference frame, $\sigma_{r}$. The mirror $B$ is co-moving. Let $\varphi$ be the constant angle between the line $A B$ and the direction $v$ of the motion. The light signal starts from Point $A$, hits the mirror in position B', is reflected, and returns to the starting point which is now in position A". (Please note, that in the case of a moving mirror, the law, according to which the incident angle equals the angle of reflection is not necessarily fulfilled in the rest inertial reference frame $\sigma_{\mathrm{r}}$, but in the moving reference frame $\sigma$ '. However, in the present context, this characteristic is of no importance.) A co-moving observer sees the light signal traveling exactly on its co-moving light path $A B$, in both directions.
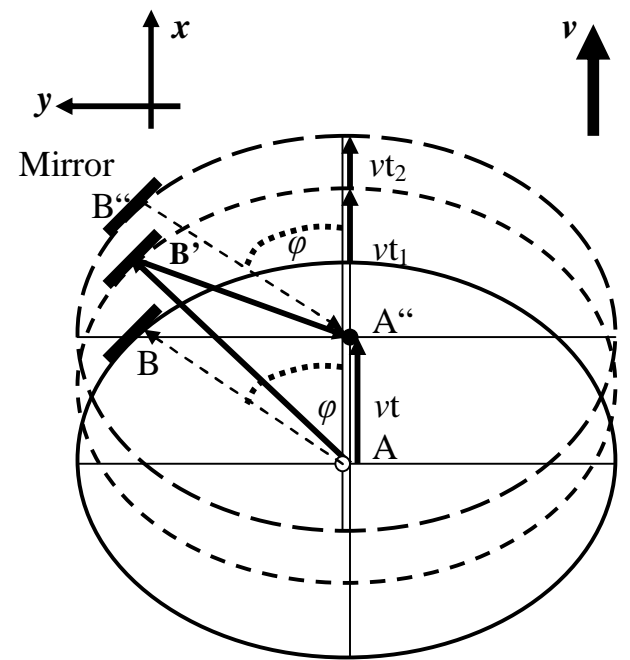

\section{Fig. 3.3. Arbitrary angle $\varphi$ between light path and direction of motion}

The light needs the time $t_{1}$ to cover the distance $A B$ '. Then, according to Euclid, the length of the path under consideration of the cross- and the length contraction and the motion from $B$ to $B^{\prime}$ is with $T_{1}=R\left(1-\beta^{2}\right)^{1 / 2} \sin (\varphi) \quad$ and $T_{2}=R\left(1-\beta^{2}\right) \cos (\varphi)$

$$
c t_{1}=\left(T_{1}^{2}+\left(T_{2}+v t_{1}\right)^{2}\right)^{1 / 2} .
$$


In a similar manner, we find the relation of the length of the return path, with the only difference that the term $v t_{1}$ changes its sign. So, we obtain

$$
c t_{2}=\left(T_{1}^{2}+\left(T_{2}-v t_{2}\right)^{2}\right)^{1 / 2} .
$$

Squaring Eq. (3.9) results in

$$
c^{2} t_{1}^{2}=T_{1}^{2}+T_{2}^{2}+2 T_{2} v t_{1}+v^{2} t_{1}^{2}
$$

This is a polynomial of degree 2 in $t_{1}$. We divide by the factor of $t_{1}^{2},\left(c^{2}-v^{2}\right)$, and draw the positive square root using the $p$-q-formula. With

$$
t_{0}=\frac{v T_{2}}{c^{2}-v^{2}}
$$

we obtain

$$
t_{1}=t_{0}+\left(t_{0}^{2}+\frac{T_{1}^{2}+T_{2}^{2}}{c^{2}-v^{2}}\right)^{1 / 2} .
$$

In the same manner as in Eq. (3.13), we find the runtime, $t_{2}$, of the return path B'A“,

$$
t_{2}=-t_{0}+\left(t_{0}^{2}+\frac{T_{1}^{2}+T_{2}^{2}}{c^{2}-v^{2}}\right)^{1 / 2} .
$$

We can simplify the expression under the square root symbol. Because of $1-\beta^{2}=\left(c^{2}-v^{2}\right) / c^{2}$, and Eq. (3.12), and by decomposing the middle term into two fractions, we obtain

$$
\begin{aligned}
& \frac{v^{2} R^{2} \cos ^{2}(\varphi)}{c^{4}}+\frac{R^{2} \cos ^{2}(\varphi)}{c^{2}}- \\
& \frac{v^{2} R^{2} \cos ^{2}(\varphi)}{c^{4}}+\frac{R^{2} \sin ^{2}(\varphi)}{c^{2}}
\end{aligned}
$$

The first and the third terms cancel each other out, and because of $\sin ^{2}(\varphi)+\cos ^{2}(\varphi)=1$, we obtain for the runtime $t_{1}$ for distance $A B^{\prime}$,

$$
t_{1}=\frac{v R\left(1-\beta^{2}\right) \cos (\varphi)}{c^{2}-v^{2}}+\frac{R}{c} .
$$

In a similar manner, we obtain from Eq. (3.10) the runtime $t_{2}$ of the distance B'A“,

$$
t_{2}=-\frac{v R\left(1-\beta^{2}\right) \cos (\varphi)}{c^{2}-v^{2}}+\frac{R}{c}
$$

Thus, the total runtime $t$ is

$$
t=t_{1}+t_{2}=2 \frac{R}{c} .
$$

Evidently, the runtime $t$ has not changed in comparison with the runtime in the rest inertial reference frame $\sigma_{r}$. This is true for each velocity $v<c$ and for each angle $\varphi$ between the light path and the direction of motion, $v$.

\section{TIME AND TIME TRANSFORMATION}

The cosmological model of the cosmic membrane theory posits that our threedimensional world is the surface of a fourdimensional sphere that expands with great velocity. This expansion causes and controls all physical processes. Therefore, time is connected with the fourth spatial dimension, specifically with the velocity of the expansion. In relativity theory, one describes this connection by the term $-c t$, i.e., not time, but the distance traveled per time unit, and, thus, a fourth spatial dimension. Nevertheless, we can use, in cosmic membrane theory, the notion space-time because the fourth spatial dimension (i.e., the radius of the cosmic balloon) changes with $V_{E} t$, where $V_{E}$ is the unknown velocity of expansion.

Time is measured via cyclical processes (clocks). In this process, baryonic matter is always involved. The speed of the cyclical processes depends (among other things) on the speed $v$ of the motion of the clock in the rest inertial reference frame. The experiments of Ives and Stilwell [25], the clock experiment of Haefele und Keating [26], and experiences with the GPS (Global Positioning System) suggest that the time unit $\Delta t$ dilates when the clock moves with speed $v$. It is

$$
\Delta t^{\prime}=\Delta t /\left(1-\beta^{2}\right)^{1 / 2} .
$$

Here, $\Delta t^{\prime}$ is the duration of a time unit (e.g. the oscillation of a pendulum) of the clock in motion, and $\Delta t$ is the duration of the time unit of the same clock, resting in the rest inertial reference frame $\sigma_{r}$. Both durations are measured in units of the rest inertial reference frame $\sigma_{\mathrm{r}}$. 
All clocks (including atomic clocks) on Earth vary in speed. The reason for this variation is that the Sun moves with relatively constant speed, $v_{S}=369 \mathrm{~km} / \mathrm{s}$, in the coordinate system of the cosmic micro-wave background radiation (CMBR) in the direction of the Virgo cluster [15], but the Earth describes an additional orbit round the Sun with a speed of $v_{E}=30 \mathrm{~km} / \mathrm{s}$.

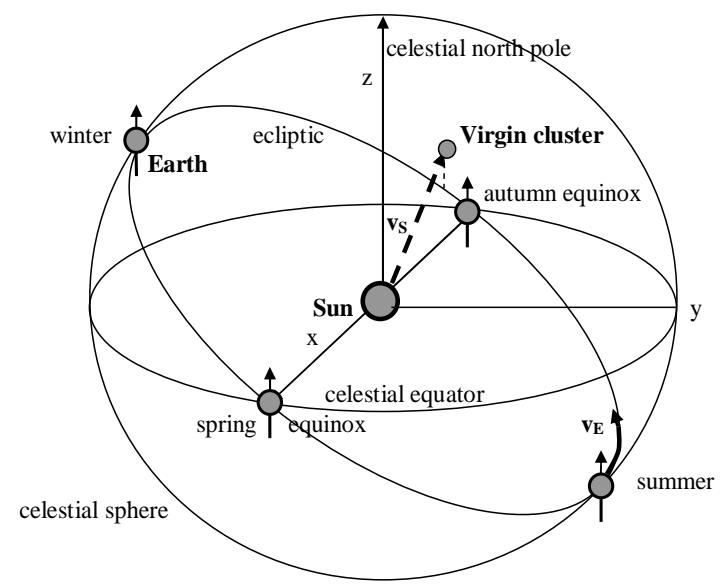

Fig. 4.1. The motion of the Sun and the orbit of the Earth

The Virgo cluster is located near the ecliptic, and close to the autumn-equinoxes in the constellation Virgo (Virgin). When the Sun stands, in autumn, in the Virgo constellation, then, seen from the Sun, the Earth stands in the Aries constellation, i.e., at the spring equinox point (see Fig. 4.1). Therefore, in summer, the velocity $v_{S}$ of the Sun and the velocity $v_{E}$ of the Earth add together, but in winter, we have to subtract $v_{E}$ from $v_{S}$. Thus, we obtain a rough modeling formula of the varying speed by the expression $v(t)=v_{S}+v_{E} \sin \left(\omega\left(t-t_{0}\right)\right)$ or $v(t)=369+30 \sin \left(\omega\left(t-t_{0}\right)\right)$. Here, $t_{0}$ is the start of spring, and $\omega=2 \pi / a$ is the angular frequency with $a$ as the duration of one year.

The runtime $t$ is constant for a light signal traveling in the rest inertial reference frame in both directions over a given distance $s$. The runtime $t$ is also independent of the direction and speed $v$ of the motion of the route $\sigma^{\prime}$. Therefore, a changing time unit $\Delta t^{\prime}$ in the moving reference frame $\sigma$ ' will cause an apparent change in the speed $c$ of light.

We denote by $\Delta t^{\prime \prime}$ the time unit at the Earth in summer, by $\Delta t^{\prime}$ the time unit in winter, and by $\Delta t$ the time unit of the rest inertial reference frame.
Then, we find with $v_{S}=369[\mathrm{~km} / \mathrm{s}]$ and $v_{E}=30$ $[\mathrm{km} / \mathrm{s}]$ and $c=299792[\mathrm{~km} / \mathrm{s}]$

$$
\Delta t^{\prime \prime}=\frac{\Delta t}{\left(1-\frac{\left(v_{S}+v_{E}\right)^{2}}{c^{2}}\right)^{1 / 2}}=\Delta t \cdot 1.00000088568,
$$

$$
\Delta t^{\prime}=\frac{\Delta t}{\left(1-\frac{\left(v_{S}-v_{E}\right)^{2}}{c^{2}}\right)^{1 / 2}}=\Delta t \cdot 1.00000063934
$$

The apparent difference of the speed of light between summer and winter is

$$
\Delta c_{\max }=\frac{\Delta t^{\prime \prime}}{\Delta t^{\prime}} c-c=73.8[\mathrm{~m} / \mathrm{s}] .
$$

This is twice the amplitude of the sinusoidal course of the apparent change in the speed of light. Therefore, the amplitude is $\Delta c=36.9[\mathrm{~m} / \mathrm{s}]$.

This hypothesis of a changing speed of light is in contradiction to the very exact measurements of Evenson and Petersen [13]. Both authors have, already in 1972, found the constancy of the speed of light up to some fractions in $[\mathrm{m} / \mathrm{s}]$.

But we can solve this contradiction. The unit of length, the meter [m], has been coupled in 1973 to the wavelength of the light that is emitted by the nuclide $86 \mathrm{Kr}$ (krypton) during the transition from State $5 d_{5}$ to State $2 p_{10}$. This fact should cause no problem, because the positions of the nodes do not change for the two superposing waves of the forward and the return path in the interferometer $[10,23]$. But Evenson and Petersen [13] have not used the distance of $1,650,763.73$ nodes of the ${ }^{86} \mathrm{Kr}$-light as the unit of length, but the wavelength $\lambda_{\mathrm{CH}}$ of a methanelaser. Barger und Hall $[27,28]$ had frozen this wavelength to a constant by comparing it with the ${ }^{86} \mathrm{Kr}$-standard of length using a Fabry-Perot interferometer. From now on, this wave length $\lambda_{\mathrm{CH} 4}$ has replaced the ${ }^{86} \mathrm{Kr}$-standard of length. The frequency of the He-Ne-laser used for the above-mentioned measurements of the speed of light was stabilized with the frequency $v_{C H}$ of the $\mathrm{CH} 4$-molecule, and measured by a comparison with a frequency standard which was coupled to the standard of time. Then, one obtains the speed of light by the simple equation $c=v_{\mathrm{CH} 4} \lambda_{\mathrm{CH} 4}$. 
By their work, Barger und Hall have set the wavelength $\lambda_{\mathrm{CH} 4}$ to a constant, but the frequency $v_{C H 4}$ of the $\mathrm{CH} 4$-molecule changes proportionally to the standard of time, as does each clock, which is supposed to also be a constant by this measuring method. This way, one obtains, after all, the correct result, i.e., the constancy of the speed of light, independent of the seasonal change in the time standard and wavelength $\lambda_{\mathrm{CH} 4}$. At the very end, this handling of time is legitimate. It stands for the geocentric time system.

To illustrate the extraordinary quantum leap in the accuracy of the measurement of the speed of light, we mention that other authors had performed measurements of the speed of light at the time of Evenson and Petersen as well. The value of Evenson and Petersen published in 1972 was $\mathrm{c}=299792.4562 \pm 0.0011 \mathrm{~km} / \mathrm{s}$, and had thus an error of only $1.1 \mathrm{~m} / \mathrm{s}$. Here are some other measurements for comparison: Kolibayev [29] found the value of $\mathrm{c}=299792.6 \pm 0.06 \mathrm{~km} / \mathrm{s}$ in 1965, Grosse [30] found the value of $\mathrm{C}=299792.5 \pm 0.05 \mathrm{~km} / \mathrm{s}$ in 1967, Bjerhammar [31] found the value of $\mathrm{C}=299792.375 \pm 0.06$ $\mathrm{km} / \mathrm{s}$ in 1972, and Guelachvili [32] found the value of $c=299792.46 \pm 0.07 \mathrm{~km} / \mathrm{s}$ in 1973 . As one can see, the errors of these four authors are between 50 and $70 \mathrm{~m} / \mathrm{s}$, i.e., they are all greater than our predicted value of $\pm 36.9 \mathrm{~m} / \mathrm{s}$, which results from the influence of the motion of the Earth.

\section{PROPOSAL OF A NEW MEASURE- MENT OF THE TWO-WAY SPEED OF LIGHT}

The inaccuracy of the clocks caused by the change in the seasons is no problem for technical and most of scientific applications because the value of $\Delta t^{\prime \prime} / \Delta t^{\prime}=1.000000123$ of the error is of the order $10^{-7}$. We propose exploiting this situation to provide evidence of the rest inertial reference frame by new measurements of the speed of light. Specifically, we propose returning to the old method of measurement of the speed of light, i.e., a method that uses a fixed light path. However, the requirements are near the limits of the feasible of today.

We propose performing, e.g., a repeated measurement of the runtime $t$ of light over a fixed course with a mirror at one end and with a total distance (bidirectional) of about 200 [km] over the time of one year. One can find in Germany a suitable course. It is the distance between the Brocken, the highest hill in the Harz mountains, and the Grosser Inselsberg, a hill in the Thuringia forest. Carl Friedrich Gauss has undertaken the triangulation of these hills already in 1820 . The distance is $106 \mathrm{~km}$. Thus, the runtime is about $\mathrm{t}=212 / 300000=0.0007$ [s], which one would have to measure with an accuracy of $5 \times 10-8$. To accomplish this, one needs a digital counter with the frequency $v=1 /(0.7 \times 10-3 \cdot 5 \times 10-8)=$ $28 \times 10^{9}$, i.e., $28 \mathrm{GHz}$. Using an economically priced cesium clock with $9.2 \mathrm{GHz}$ and a digital counter with an adequate speed, one has to repeat each single measurement 16 times to reduce the size of the mean quadratic error to a value of $5 \times 10^{-8}$. The length $s$ of the course must be constant in the order of $5 \times 10^{-8}$, too, i.e., within 5 [mm]. However, there is no need to measure the length of the course with that accuracy, because we are not interested in measuring the absolute value of the speed of light, but only the seasonal fluctuations.

From our point of view, one can enumerate other sources of measurement error:

- Changes in the course $\mathrm{s}$ by tectonic displacements. One could eliminate a linear trend of the change by means of regression analysis.

- Changes in the course $s$ by gravitationally caused tectonic displacements, i.e., by the fluctuating influence of the Sun and the Moon. Here, one could eliminate the cyclical influence by Fourier analysis.

- Changes in the course $s$ by seasonal fluctuations of the temperature: The seasonal fluctuations of the temperature can influence the position of the laser and the mirror by thermal expansion of buildings. Thermal expansion fluctuates in the same rhythm as the expected effect of the speed of light. Therefore, one cannot eliminate this influence by Fourier analysis. Here, it would be important to record these possible errors using precise measurements of the distance between laser and mirror with reference to fixedpoints in the near environment.

- The atmospheric air pressure, the temperature of the air, and the humidity of the air have an influence on the measurement. Near the laser and the mirror, it is important to record the atmospheric air pressure with an accuracy of 1 [mbar], the temperature with an accuracy of $1\left[{ }^{\circ} \mathrm{C}\right]$, and the humidity with 
an accuracy of $5 \%$. Using these measures, one can eliminate the fluctuating influence on the speed of light. The errors are in the order of $10^{-7}$. That means, one should repeat the single measurement at least 4 times after short time intervals.

One can automate the measuring process. Therefore, thousands of individual measurements per hour are possible when visibility is good. So, the possibility exists to show the expected effect after one year, if it exists.

\section{ONE-WAY SPEED OF LIGHT}

The measurement of the one-way speed of light is a problem in a moving inertial reference frame. The one-way speed of light means the measurement of the speed of light over a single distance without reflection of the beam of light and its return to the starting point. Such a measurement of the one-way speed of light needs two synchronized clocks, one clock at the starting point and another clock at the arrival point. However, synchronization is the issue. We will demonstrate, in agreement with the SR $[33,34,35,36]$, that a measurement of the oneway speed of light is not possible because of the relativistic time effect of clocks.

The intuitive expectation is that the relative speed of light should be smaller than $c$ if the motion of the moving inertial reference frame and that of the beam of light are parallel. If the motion is antiparallel, the speed of the light should be greater than $c$. But this intuitive expectation will be disappointed. The reason is that the relativistic effects of the time transformation of moving clocks hide the proper motion of the moving inertial reference frame nearly perfectly. Even a very slow transport of the second clock $[33,36]$ away from the point of synchronization does not solve the problem.

In 2011, the OPERA group [37] published a paper on the one-way measurement of the velocity of neutrinos. The published results were the outcome of three years of measurement in the Neutrino Laboratory of the Gran Sasso massif. The working group has measured the velocity of neutrinos with a significant deviation from the speed of light. The relative deviation was specified as $(\mathrm{v}-\mathrm{c}) / \mathrm{c}=2.48 \times 10-5$. This amount corresponds to an absolute increase of $7.5 \mathrm{~km} / \mathrm{s}$. The mean error of the measurement of the speed of light given by Evenson [13] is about $\pm 1.1 \mathrm{~m} / \mathrm{s}$. At that time, the paper of the OPERA group provoked numerous reactions, because, by the understanding of the theory of relativity, no speed can be greater than $\mathrm{c}$. We do not want to continue here the discussion, but we wanted to illustrate the great interest of science in the subject speed of light, especially the one-way speed.

The relativistic time dilation is in $\mathrm{CM}$ the same as in the Special Relativity (see Section 4, Equation 4.1). Eq. (4.1) says that the duration of any process increases if the process takes place not in the rest inertial frame $\sigma_{r}$ but in a moving inertial frame $\sigma$ '. Time t' of the duration of a process is longer (measured in $\sigma_{\mathrm{r}}$-scales) in the moving inertial frame $\sigma$ ' than in the rest frame $\sigma_{\mathrm{r}}$. Here, both durations are measured in units of the rest inertial reference frame $\sigma_{\mathrm{r}}$. Let vector $v$ be the speed of the moving reference frame $\sigma^{\prime}$ in $\sigma_{\mathrm{r}}$. Now consider a clock moving with speed $u$ inside the moving reference frame $\sigma$ '. Both vectors, $u$ and $v$, are given in in $\sigma_{r}$-scale units. Now, $d t^{\prime}$ denotes the duration of a time unit of a clock (e.g. the oscillation of a pendulum) resting in the moving reference frame $\sigma$, and $d t$ " the duration of a time unit of a clock with speed $u$ relative to $\sigma^{\prime}$, both quantities, $d t^{\prime}$ and $d t^{\prime \prime}$ in $\sigma_{\mathrm{r}}$-scale units. We obtain

$$
d t=d t^{\prime \prime}\left(1-(v+u)^{2} / c^{2}\right)^{1 / 2}
$$

Now we replace dt by dt' according to Eq. (4.1), i.e.

$$
d t=d t^{\prime}\left(1-v^{2} / c^{2}\right)^{1 / 2}
$$

We obtain

$$
d t^{\prime \prime}=\left(1-v^{2} / c^{2}\right)^{1 / 2} /\left(1-\frac{(v+u)^{2}}{c^{2}}\right)^{1 / 2} \cdot d t^{\prime}
$$

If speed $v$ of frame $\sigma^{\prime}$ inside the rest frame $\sigma_{r}$ is smaller with respect to the speed of light $(V<<c)$ and $u$ is much smaller than $v(u<v)$, then Eq. (6.2) simplifies. We find

$$
d t^{\prime \prime}=\left(1+\frac{(v+u)^{2}}{2 c^{2}}\right) \cdot\left(1-\frac{v^{2}}{2 c^{2}}\right) \cdot d t^{\prime}
$$

If the vectors $v$ and $u$ are parallel, then $(v+u)^{2}=$ $v^{2}+2 v u+u^{2}$ holds. Because we have assumed 
that $u$ is much smaller than $v$ we can neglect $u^{2}$. We find the approximation $(v+u)^{2} \approx v^{2}+2 v u$. Eq. (6.4) simplifies further to

$$
d t^{\prime \prime}=\left(1+\frac{v u}{c^{2}}\right) d t^{\prime}
$$

(If the vectors $v$ and $u$ have opposite direction then $(v-u)^{2}=v^{2}-2 v u+u^{2}$. Similarly to Eq. (6.5) we find $d t^{\prime \prime}=\left(1-v u / c^{2}\right) d t^{\prime}$.)

Eq. (6.5) describes the change in the duration of the time units of two clocks with different speed relative to the rest inertial frame $\sigma_{\mathrm{r}}$ in scale units of $\sigma_{\mathrm{r}}$. The duration of the time units (seconds) of clock t" are longer than the duration of the time units of clock t'. That means, otherwise, that a time $t$ measured by clock t" has a smaller numerical amount than that of $t$ '. Therefore, time $t$ measured by clock $\mathrm{t}$ " is shorter than the same time $t$ measured by clock t'. Clock t" is late compared with clock t'. That means, if we want to compute the time t" in scale units of the moving inertial frame $\sigma$ ', we have to integrate with the inverse transformation factor of Eq. (6.5), i.e., with transformation factor $1 /\left(1+v u / c^{2}\right)$, or, because of $v<<c$ and $u<<v$, with factor $\left(1-v u / c^{2}\right)$.

Now, we consider the movement of a clock from coordinate $x_{1}^{\prime}$ of frame $\sigma^{\prime}$ to coordinate $x_{2}^{\prime}$ with a constant low speed $u(u<<v)$. We assume the vectors $v$ and $u$ to be parallel. We replace dt' by the fraction $d x^{\prime} / u$, where $d x^{\prime}$ is a differentially small distance in the moving reference frame $\sigma^{\prime}$. We integrate Eq. (6.5) using the above mentioned transformation factor $\left(1-v u / c^{2}\right)$.

$$
t^{\prime \prime}=\int_{x_{1}^{\prime}}^{x_{2}^{\prime}}\left(\frac{1}{u}-\frac{v}{c^{2}}\right) d x^{\prime} .
$$

With $\mathrm{t}^{\prime}=\left(\mathrm{x}_{2}^{\prime}-\mathrm{x}_{1}^{\prime}\right) / \mathrm{u}$ we obtain

$$
t^{\prime \prime}=t^{\prime}-\frac{v\left(x_{2}^{\prime}-x_{1}^{\prime}\right)}{c^{2}}
$$

Here $s^{\prime}=\left(x^{\prime}{ }_{2}-x^{\prime}\right)$ is the distance of the movement in the moving inertial frame $\sigma$ '. The reading of the clock at position $x_{2}^{\prime}$ is by $\Delta t^{\prime \prime}=v s^{\prime} / c^{2}$ less than that of the clock at position $\mathrm{x}^{\prime}{ }_{1}$. Otherwise, if $u$ and $v$ have opposite directions, the reading of the clock at $x_{2}^{\prime}$ is by $\Delta \mathrm{t}^{\prime \prime}=v \mathrm{~s}^{\prime} / \mathrm{c}^{2}$ greater than that of the clock at position $\mathrm{x}^{\prime}{ }_{1}$.

It is not shown here, but this result does not depend on the path of movement itself. Only the starting line $x_{1}^{\prime}$ and the ending line $x_{2}^{\prime}$ of the movement are of interest. If vector $u$ is not parallel or antiparallel to vector $v$, we have to replace the product $v \cdot d x^{\prime}$ by the scalar vector product $\vec{v} \cdot d \vec{x}^{\prime}$. This means that only those components of the path $s^{\prime}$ are of interest that are parallel or antiparallel to the speed $v$ of the comoving reference frame.

Now, we return to the example of measuring the one-way speed of light. Two clocks, clock $_{1}$ and clock $_{2}$, are synchronized at the coordinate $x_{1}$ ' of the moving inertial frame $\sigma$ '. Both readings are $\mathrm{t}_{0}$ '. Now, clock $_{2}$ is moved with constant low speed $u$ to the new coordinate $x_{2}$ '. The reading of clock is $t_{1}{ }^{\prime}$, that of clock $_{2}$ is $t 1^{\prime}-\left(x_{2}^{\prime}-x_{1}{ }^{\prime}\right) v / c^{2}$. We start a beam of light from coordinate $\mathrm{x}_{1}^{\prime}$ to $\mathrm{x}_{2}^{\prime}$. Neglecting terms with $v^{2} / c^{2}$ and higher, the beam of light passes the distance $s^{\prime}=\left(x_{2}{ }^{\prime}-x_{1}{ }^{\prime}\right)$ needing the time interval $\Delta t_{1}{ }^{\prime}=s^{\prime} / c$ or $\Delta t_{1}{ }^{\prime}=\left(x_{2}{ }^{\prime}-x_{1}{ }^{\prime}\right) / c$. The reading of clock $_{1}$ is $t_{1}{ }^{\prime}+\Delta t_{1}$ ', that of clock ${ }_{2}$ is $t_{1}{ }^{\prime}+\Delta t 1^{\prime}-\left(x_{2}{ }^{\prime}-x_{1}{ }^{\prime}\right) v / c^{2}$.

But the moving inertial frame $\sigma$ ' has meanwhile moved on by the small distance $\Delta x^{\prime}=\Delta t_{1}^{\prime} \cdot v$. The beam of light needs for this additional distance the time $\Delta t_{2}^{\prime}=\Delta x^{\prime} / c$ or $\Delta t_{2}^{\prime}=\left(x_{2}^{\prime}-x_{1}^{\prime}\right) \quad v / c^{2}$. The reading of clock ${ }_{1}$ is now $t_{1}{ }^{\prime}+\Delta t_{1}{ }^{\prime}+\Delta t_{2}$ ', that of clock $_{2}$ is $t_{1}{ }^{\prime}+\Delta t_{1}{ }^{\prime}+\Delta t_{2}{ }^{\prime}-\left(x_{2}{ }^{\prime}-x_{1}{ }^{\prime}\right) \quad v / c^{2}$ or $t_{2}{ }^{\prime}=t_{1}{ }^{\prime}+\Delta t_{1}{ }^{\prime}$. The co-moving observer registers the arrival of the beam at the coordinate $x_{2}{ }^{\prime}$ at time $t_{2}{ }^{\prime}$. His logical conclusion is in accordance with the SR: Light travels within the time interval $\Delta t_{1}{ }^{\prime}=\left(x_{2}{ }^{\prime}-x_{1}{ }^{\prime}\right) / c$ over the distance $s^{\prime}=\left(x_{2}{ }^{\prime}-x_{1}{ }^{\prime}\right)$, consequently the one-way speed of light is $c$, regardless of the speed $v$ of the moving inertial frame $\sigma^{\prime}$. For this result, it was sufficient to assume the existence of the rest inertial reference frame and the transformation of time.

\section{RESULTS AND DISCUSSION}

Although the speed of light in the vacuum is constant, it can be measured on Earth only with a systematic error of $\pm 36.9 \mathrm{~m} / \mathrm{s}$. The reason is that the flow of time of the clocks on the Earth is not constant. The flow of time depends on the motion of the Earth in absolute space, i.e., on the motion of the Earth around the moving Sun. We predict, for new measurements of the speed of light, a sine-shaped yearly variation with an 
amplitude of $36.9 \mathrm{~m} / \mathrm{s}$ and a maximum reached in summer. Evenson and Petersen have replaced the measurement of the distance by a measurement of a frequency (and thus by a second measurement of time). The consequence is that a change in the time flow has no further influence on the measurement of the velocity. Naturally, this handling of the time is legitimate, but it uses the geocentric time system.

The special problem of measuring the one-way speed of light was treated in a moving inertial reference frame. Measuring the one-way speed of light implies the measurement of the speed of light over a single distance without reflection of the beam and its return to the starting point. The issue is the synchronization of the clocks. A correct synchronization can be achieved in the rest inertial reference frame only by a very slow movement of the clocks towards different directions. However, when the synchronization is performed in a moving inertial reference frame, the readings of the clocks change, so that one measures always the speed of light as $\mathrm{c}$, independent of the velocity $v$ of the moving frame relative to the rest frame. Although we had to add the speed $v$ of the moving inertial reference frame in a Galilean manner, the time transformation hides this fact nearly perfectly. A co-moving observer registers the arrival of the light signal after a time interval which suggests that the speed of light is $c$, and not $c+v$ or $c-v$ in the cases of antiparallel or parallel motion of light and reference frame. For this result, it is sufficient that we accept the existence of a rest inertial reference frame and the transformation of time as given in the SR. Otherwise, for the measurements of the time of flight of neutrinos or gravitational waves one needs accurate time synchronization. That means, one has not only to consider the distance of the clocks, but also the orientation of the connecting line with respect to the movement of the Earth relative to the rest frame.

Moving bodies experience both, a length contraction and a cross contraction. The twin paradox does not appear because of the real nature of the time transformation. Each twin has its own subjective time that depends on its motion. However, to differentiate between absolute or relative motion, we cannot use the atomic clock experiment of Haefele and Keating because we had to bring together the clocks. Only this way, we can compare the readings of the clocks. So, we obtain a closed contour, and the linear effect of the absolute motion disappears. The quadratic effect is the same as in the SR.

The main aim of this paper is to show that the speed of light, $c$, is constant in each inertial reference frame also under the paradigm of an absolute space. The existence of an absolute space became probable by the discovery of the cosmic microwave background radiation by Wilson and Penzias. In the special case of oneway measurement of the speed of light, we can show that speed $c$ is measured as a constant again, in spite of its anisotropy in a moving inertial frame of reference.

Our 4D cosmological model with four spatial dimensions and extra time was constructed such that we conserve a high level of conformity between classical SR, GR, and our model. This means that our model is, in its present stage, not suitable to explain any connection between gravity and high energy physics, i.e., gravity and QED or QCD. We refer to our former papers $[4,7,8,9]$ for more information on our membrane model. The model is the direct consequence of the supposition of an absolute space. In [4], we showed that a 3D elastic membrane stretched in a 4D bulk space has, under the action of a central load, exactly the curvature that is required by Newton's law of gravitational attraction. In [9], we obtained the same effects as Puthoff [11] on the speed of light and the mass of particles, depending on the strength of the gravitational field. Therefore, using these effects, the direct influence of time is replaced by an indirect influence. The gravitational effects on the speed of light and the mass of particles explain experiments and observations of the GR with the same exactness in the context of the $\mathrm{CM}$ model.

The conformity between the cosmic membrane theory and the special theory of relativity (SR) is about $90 \%$. The only important difference is that membrane theory introduces a cross-contraction of moving bodies. Conformity between General Relativity (GR) and CM is also nearly $100 \%$. This conformity concerns light bending, time delay of radar signals, gravitational red shift, gravitational time dilation of clocks, gravitational waves, and red shift caused by the expansion of the Universe. One difference is the change of the speed of light and the change of mass in gravitational fields. But here, with reference to Puthoff's "Polarizable Vacuum approach to GR" [11], CM and GR are also in agreement. 
The Michelson-Morley experiment posits that the total light path (the path to the mirror and back) does not change if one rotates the interferometer. If one supposes a length contraction in direction of the relative motion (and no cross contraction) as done by FitzGerald and Lorentz, then the length contraction must be naturally that of FitzGerald (1884) and Lorentz (1894), that is $\left(1-v^{2} / c^{2}\right)^{1 / 2}$. Otherwise, if one supposes both contractions, the cross and the length contraction, the ratio of length to cross contraction must be $\left(1-\mathrm{v}^{2} / \mathrm{c}^{2}\right)^{1 / 2}$. Because we had found by other considerations the cross contraction with the contraction factor $\left(1-v^{2} / c^{2}\right)^{1 / 2}$, the length contraction must inevitably be $\left(1-\mathrm{v}^{2} / \mathrm{c}^{2}\right)$ to describe correctly the experiment.

This is the classical case that one and the same experiment (the Michelson-Morley experiment) can be described correctly by two different transformations. Both variants are correct. Which variant one should prefer in future will depend on further experiments. Perhaps the proposed experiment concerning new measurements of the speed of light could be helpful.

In principle, the way in which FitzGerald und Lorentz found length contraction is logical and consequent. The cross contraction in our theory was not only introduced to explain the MichelsonMorley experiment, but also the equilibrium of attracting and repulsing forces between two moving charged particles at parallel trajectories. Length contraction is then a logical consequence. In nearly all cases, it does not matter whether one uses the FitzGerald-Lorentz transformation or the transformation with cross and length contraction. One can explain with the same exactness and with both transformations not only the Michelson-Morley experiment, but also, for example, Maxwell's equations, Fresnel's drag coefficient, Fizeau's experiment, aberration and Airy's experiment, the atomic clock experiment of Haefele and Keating, the TroutonNoble experiment, and, last not least, the Thomas precession of the spinning electron. Other physical reasons of the introduction of the cross contraction of moving bodies are (1) the above-mentioned decrease of the cross section of accelerated particles, and (2) the energy content of a moving crystal. Onoochin and von Weber [10] showed that the energy content of a moving crystal does not decrease only when one supposes both cross and length contraction. Naturally, special relativity with extra dimensions is an interesting theoretical area [compare e.g. 38], but in our case of the reintroduction of the absolute space, we needed only the classical SR with the addition of a cross contraction.

As far as the Compton wavelength is concerned, one could suspect that the cross contraction could be in contradiction to Compton's theory. The formula of the Compton wavelength contains the speed $v$ of the particle and its relativistic mass $m$. However, in this respect, there is no difference between SR and CM. One proof is the correct calculation of the Thomas factor as $1 / 2$ of the spinning electron with and without cross contraction. Furthermore, we use the relativistic increase of the mass in the sense of Puthoff's Polarizable-Vacuum approach to GR [compare 9]. The starting point is the relativistic square of the energy, $E^{2}=\left(m c^{2}\right)^{2}+(p c)^{2}$. We have found the same effects of speed, mass and time that Puthoff published in 2002 [11]. This proves that our theory is conform with GR. Therefore, we trust that the introduction of the cross contraction is not seen just as a curiosity, but as an essential step of improvement of all models that use an absolute space. Supposing that neutrinos travel with vacuum speed of light, our citation of the OPERA experiment [37] makes sense. This experiment is a typical case of a one-way measurement of the speed of light. We argue, however, in contradiction to the results of the OPERA group, that one cannot find any deviation from the speed of light. The different readings of the clocks hide the different distances, over the course of one year,that neutrinos have to travel in absolute space. The result of the one-way measurement of the speed does not differ from the two-way measurement, that is, there is no possibility to measure or to prove the anisotropy of the speed of light in a moving inertial reference frame.

With our proposal of a new measurement of the vacuum speed of light, we do not doubt the very precise measurements of Evenson et al. [13]. We are not interested in further improving of the high exactness of Evenson's result, but in the verification of the existence of an absolute space. One way to reach this aim could be to prove that the speed of light changes with the seasons if one returns to the old method of measurement. (Besides, it is true that Carl Friedrich Gauss has triangulated the distance of several hills in Germany not only to find the distances, but to discover deviations from the flatness of the space we live in.)

Naturally, there remain open questions. We treat $\mathrm{SR}$ in 4D with extra time. Velev [38] treats 
transformations with extra dimensions. Cabbolet $[39,40]$ treats $S R$ in $5 \mathrm{D}$, but with the aim to identify laws that would govern the universe if antimatter having rest mass would be repulsed by the gravitational field of the earth. We assume ordinary matter and do not consider antigravity, although in the special case of the collapse of a red giant gravitational repulsion could be possible.

The following part of the discussion does not only concern this paper and its results, but is also related with the background of our cosmological model. We work with a special 4D model with extra time. In this model, the fourth dimension appears (1) as the expanding radius, $\mathrm{w}=\mathrm{V}_{\mathrm{E}} \mathrm{t}$, with speed $V_{E}$ of expansion. But the expansion does not play any role that would concern gravity and its effects. The fourth dimension, w, is much more important as depth of space, that is the deviation of the curvature of space from the curvature of the exact 4D sphere. If we consider small pieces of space, the global curvature of space becomes minimal, and only that part of the curvature remains that is caused locally by the agglomeration of masses. Here, the homogeneous vector field, acting from the fourth dimension, plays the leading part [4].

Wesson [41] discusses brane world models in different dimensions with the aim of the unification of the gravitational field and its source, the matter. Starting from the 5D Ricci equations, Wesson derives Einstein's 4D field equations of GR with a source. The equations describe gravity, electromagnetism and the scalar field. Interesting for us is, besides the general overview of the landscape of brane worlds, the discussion concerning the cosmological constant. In our model, the cosmological constant is closely connected with the unknown density of the membrane. In contrast to Kaluza [2] or Wesson [41], we do not try to connect gravity with electromagnetism and quantum theory, but restrict ourselves to classical effects of the SR and GR and their tests.

Boehmer, Harko, and Lobo [42] parametrize classes of brane wold models, extract terms containing those parameters that could lead to deviations from the GR, and compare these deviations with the known experimental data of several tests of GR. Already two parameters stress the limited precision of the experimental data. The results are rough limits for the chosen parameters. But the chosen method is convincing. We calculated the tension of the membrane of our model in a similar manner. In our case as well, the used data of light bending by the Sun delivered a value with a very large error bar. Therefore, we decided to compute the tension by a direct connection to the GR using Feynman's excess radius.

\section{CONCLUSIONS}

This article shows differences between CM and $\mathrm{SR}$. Some of the discussed differences concern the interpretation of the basic physical principles but lead to the same results as the SR. However, in some cases we find slightly differing results. One example is the prediction of a sine-shaped yearly variation of the speed of light in the order of $10^{-7}$. For practical purposes, this variation is not essential, and the cause is not a real alteration in the speed of light, but a problem of the correct choice of the used time system.

The introduction of a cross contraction of moving bodies is the only essential difference between $\mathrm{CM}$ and SR. There is no direct proof that the cross contraction exists, but there exists also no evidence that the length contraction really exists. However, logical arguments in the frame of the $\mathrm{CM}$ sustain the introduction of cross contraction in addition to length contraction. One argument is the constancy of each closed light path independent of the velocity $v$ of a moving inertial reference frame with respect to the rest frame. Another argument is the explanation of the decrease in the cross section of moving particles.

Our derivation of the equations of the one-way speed of light in Section 6 neglects all quadratic and higher terms of $v^{2} / c^{2}$. Therefore, it would be interesting to investigate the influence of the neglected terms. Perhaps, one still finds a small dependency of the one-way speed of light on the velocity of the moving reference frame. This could offer the possibility of an experimental proof. However, we would expect only a small effect of the time as described in Section 4, i.e., a dependence on the choice of the proper time system.

Therefore, the focus of our future investigations should be to find more similarities and conformities between the theories in the sense of $\mathrm{H}$. Puthoff. On can suppose that, from a higher point of view the SR, the GR and related theories as the $\mathrm{CM}$ are only projections of a more comprehensive theory. 


\section{ACKNOWLEDGEMENTS}

We thank all peers that were involved in the peer reviewing process of this paper for their courtesy, helping advice and criticisms. We believe that the discussion helped improve the quality of this article. Further, we thank the editorial team of the PSI journal for its patience and administrative moderation.

\section{COMPETING INTERESTS}

Authors have declared that no competing interests exist.

\section{REFERENCES}

1. Penzias AA, Wilson RW. A measurement of excess antenna temperature at 4080 Mc/s. ApJ. 1965;142:419-421.

Available:http://dx.doi.org/10.1086/148307

2. Kaluza T. Zum unitätsproblem der physik, sitzungsberichte der Preussischen Akademie der Wissenschaften. 1921;966: 972.German.

Available:http://homepage.uibk.ac.at/ c70 5204/pdf/kaluza-1921.pdf

3. Thorne $\mathrm{KS}$, Price $\mathrm{RH}$, Macdonald $\mathrm{DA}$, editors. Black Holes: The membrane paradigm. Yale University Press; 1986.

4. Von Weber S, von Eye A. Multiple weighted regression analysis of the curvature of a 3D Brane in a 4D Bulk Space under a Homogeneous Vector Field. Inter Stat; 2010. Available:http://interstat.statjournals.net/YE AR/2010/articles/1007003.pdf

5. Ford LH. Inflation driven by a vector field. Phys. Rev. 1989;D40:967-972. Available:http://www.ncbi.nlm.nih.gov/pub med/10011903

6. Gogberashvili M. Brane gravity from bulk vector field. Phys. Lett. 2003;B553:284288.

Available:http://www.sciencedirect.com/sci ence/article/pii/S0370269302032082

7. Von Weber S, von Eye A. Monte Carlo study of vector field-induced dark matter in a spiral galaxy. Inter Stat; 2011. Available:http://interstat.statiournals.net/YE AR/2011/articles/1108002.pdf

8. Von Weber S, von Eye A. Error analysis of simulated Einstein rings under the membrane paradigm. Inter Stat; 2013. Available:http://interstat.statjournals.net/YE AR/2013/articles/1310001.pdf
9. Von Weber S, von Eye A. Geodetic precession under the paradigm of a cosmic membrane. Phys. Sci. Int. J. 2016; 10(4):1-14.

Available:http://www.sciencedomain.org/ab stract/14928

10. Onoochin V, vonWeber S. On the size of moving rigid bodies determined from conditions of equilibrium of ions in a crystalline lattice. In Einstein and Poincaré: The Physical Vacuum. Dvoeglazov VV, editor. Apeiron Montreal; 2006

ISBN0-9732911-3-3

Available:https://de.scribd.com/document/2 74516229/Einstein-and-Poincare-the-

Phys-Valeri-v-Dvoeglazov-Editor

11. Puthoff HE. Polarizable-Vacuum approach to GR. Found. of Physics. 2002;32(6):1-24. Available:https://arxiv.org/ftp/grqc/papers/9 909/9909037.pdf

12. Ciufolini I. Dragging of inertial frames. Nature. 2007;449:41-47.

Available:http://www.nature.com/nature/jou rnal/v449/n7158/abs/nature06071.html

13. Evenson KM, et al. Speed of light from direct frequency and wavelength measurements of the methane-stabilized laser. Phys. Rev. Lett. 1972;29(19):134649.

Available:https://dx.doi.org/10.1103\%2FPh ysRevLett.29.1346

14. Naselsky P, Novikov D, Novikov I. The physics of cosmic microwave background. Cambridge University Press; 2006.

15. Kogut $A$, et al. Dipole Anisotropy in the COBE differential microwave radiometers first-year sky maps. Astrophys. J. 1993; 419:1-6.

Available:http://arxiv.org/abs/astroph/9312 $\underline{056}$

16. Hinshaw $G$, et al. Five-Year wilkinson microwave anisotropy probe (WMAP1). Astrophys. $\quad \mathrm{J} ; 2008$. Available:http://arxiv.org/abs/0803.0547v2

17. Einstein A. On the Influence of gravitation on the propagation of light. Ann. D. Phys. 1911;35:898-908.

Available:http://www.relativitycalculator.co $\mathrm{m} / \mathrm{pdfs} /$ On the influence of Gravitation 0 $\mathrm{n}$ the Propagation of Light English.pdf

18. Bruckman W, Esteban EP. An alternative calculation of light bending and time delay by a gravitational field. Am. J.Phys.1993; 61(8):750.

Available:http://dx.doi.org/10.1119/1.1715

19. Albrecht A, Magueijo J. A time varying speed of light as a solution to cosmological 
puzzles. Phys. Rev. 1999;D59:043516 Available:arXiv:astro-ph/9811018v2

20. Magueijo J. New varying speed of light theories. Rept. Prog. Phys. 2003;66(11): 2025.

Available:http://arxiv.org/abs/astroph/0305 457

21. Jovićević S. On vacuum dispersion and light velocity. Phys. Essays. 2005;18:518. Available:http://physicsessays.org/browsejournal-2/prhtml

22. Ellis GFR. Note on varying speed of light cosmologies, Gen. Rel. Grav. 2007;39(4): 511-520.

Available:http://arxiv.org/abs/astroph/0703 $\underline{751}$

23. Müller $H$, Herrmann $S$, Braxmaier C, Schiller S, Peters A. Modern michelsonmorley experiment using cryogenic optical resonators. Phys. Rev.Lett. 2003; 91:020401.

Available:arXiv:physics/0305117

24. Joos G. Theoretical Physics. 15th edition. AULA-Verlag Wiesbaden;1989. German.

25. Ives HE, Stilwell GR. An Experimental Study of the Rate of a Moving Atomic Clock. J. Opt.Soc. Am. 1938;28:215-226. Available:https://www.researchgate.net/pu blication/249333957 An Experimental St udy of the Rate of a Moving Atomic Cl ock

26. Haefele JC, Keating R. Around-the-world atomic clocks. Predicted Relativistic Time Gains. Science. 1972;177:166-170. Available:http://www.sciencemag.org/conte nt/177/4044/168.abstract

27. Barger RL, Hall JL. Pressure shift and broadening of methane line at $3.39 \mu$ studied by laser-saturated molecular absorption. Phys. Rev. Lett. 1969;22(4). DOI: 10.1103/PhysRevLett.22.4

28. Barger, RL, Hall JL. Wavelength of the $3.39 \mu \mathrm{m}$ laser saturated absorption line of methane, Science. 1972;177(4044):168170.

Available:http://science.sciencemag.org/co ntent/177/4044/168

29. Kolibayev VA. Determination of the velocity of light from measurements with (pulsed) light rangefinders on control bases. Geodesy and Aerophotography. 1965;3:228-230, translated for the American Geophysical Union, cited in "The Atomic Constants, Light, and Time by BARRY SETTERFIELD and TREVOR NORMAN August 1987".
Available:http://www.setterfield.org/report/r eport.html

30. Grosse H. Geodimeter-2A-Messungen in Basis vergro sserungsnetzen. Nachrichten Karten-und-Vermessungwesen, Ser. I, 35, 93-106 (1967) cited in "The Atomic Constants, Light, and Time by BARRY SETTERFIELD and TREVOR NORMAN;1987.

Available:http://www.setterfield.org/report/r eport.html

31. Bjerhammar A. A determination of the velocity of light using the twin super heterodyne principle. Tellus. 1972; 24(5):481-495.

Available:http://onlinelibrary.wiley.com/doi/ 10.1111/j.2153-3490.1972.tb01574.x/epdf

32. Guelachvili G. Spectrométrie de Fourier sous vide à 100000 points. Application à la mesure absolue de nombres d'ondes et à une nouvelle détermination de $c$. Thèse d'État Université Paris-Sud, Orsay, (1973) cited in "The Atomic Constants, Light, and Time by BARRY SETTERFIELD and TREVOR NORMAN August 1987". Available:http://www.setterfield.org/report/r eport.html

33. Mansouri R, Sexl RU. A test theory of special relativity. I: Simultaneity and clock synchronization. General Relat. Gravit. 1977;8(7):497-513.

DOI:10.1007/BF00762635

34. Will CM. Clock synchronization and isotropy of the one-way speed of light. Phys. Rev. D. 1992;45(2):403. DOI:http://dx.doi.org/10.1103/PhysRevD.4 5.403

35. Yuan-Zhong Zhang. Test theories of special relativity. General Relat. Gravit. 1995;27(5):475-493.

DOI:10.1007/BF02105074

36. Spavieri G, et al. Can the one-way speed of light be used for detection of violations of the relativity principle? Phys. Lett. A. 2012;376:795-797.

DOI:10.1016/j.physleta.2012.01.010

37. Adam $\mathrm{T}$, et al. Measurement of the neutrino velocity with the OPERA detector in the CNGS beam; 2011 Available:http://arxiv.org/ftp/arxiv/papers/1 109/1109.4897.pdf

38. Velev M. Relativistic mechanics in multiple time dimensions. Physics Essays. 2012; 25(3):403-438.

39. Cabbolet MJTF. Elementary process theory: A formal axiomatic system with a potential application as a foundational 
framework for physics supporting gravitational repulsion of matter and antimatter. Ann. Phys. (Berlin). 2010;522, 699-738.

40. Cabbolet MJTF. A 5D categorical model of the elementary process theory incorporating special relativity. Available:https://philpapers.org/archive/CA BROS.pdf
41. Wesson PS. The geometrical unification of gravity with its source. Gen. Rel. Grav. 2008;40:1353.

Available:http://link.springer.com/article/10. 1007/s10714-008-0610-z

42. Boehmer CG, Harko T, Lobo FSN. Solar system tests of brane world models. Class. Quant. Grav. 2008;25:045015. Available:https://arxiv.org/abs/0801.1375

(c) 2017 Weber and Eye; This is an Open Access article distributed under the terms of the Creative Commons Attribution License (http://creativecommons.org/licenses/by/4.0), which permits unrestricted use, distribution, and reproduction in any medium, provided the original work is properly cited.

Peer-review history:

The peer review history for this paper can be accessed here: http://sciencedomain.org/review-history/19837 